\title{
Status of the PolLux STXM Beamline.
}

Benjamin Watts $^{1, *}$, Simone Finizio ${ }^{1}$, Katharina Witte ${ }^{1}$, Manuel Langer ${ }^{1}$, Sina Mayr $^{1}$, Sebastian Wintz ${ }^{1}$, Blagoj Sarafimov ${ }^{1}$ and Jörg Raabe ${ }^{1}$

1. Swiss Light Source, Paul Scherrer Institute, 5232 Villigen-PSI, Switzerland.

* Corresponding author, benjamin.watts@psi.ch

The PolLux scanning transmission soft X-ray microscopy (STXM) beamline [1-3] has been operating at the Swiss Light Source (SLS) since 2006. With a photon energy range of 270 to $1600 \mathrm{eV}$ (from the C K-edge up to the $\mathrm{Al} \mathrm{K-edge),} \mathrm{experiments} \mathrm{at} \mathrm{PolLux} \mathrm{have} \mathrm{tended} \mathrm{to} \mathrm{focus} \mathrm{on} \mathrm{organic} \mathrm{electronic}$ materials,[4-8] magnetism of the transition metals (Fe, Co and $\mathrm{Ni}$ ) [9-13] and studies of the environment [14-17]. PolLux has seen many upgrades over the years and we continue to develop both the hardware and software to enable cutting-edge science by our user-groups. Cleanliness of the beamline optics and endstation are also a priority at PolLux and our carbon contamination removal and prevention strategies have been recently reviewed.[18]

PolLux is controlled by the Pixelator software,[19] which has been developed in collaboration with the Max-Planck-Institut für Intelligente Systeme and Semafor Informatik \& Energie AG for reliable, efficient and user-friendly operation of experiments. Many hardware interface modules have been implemented for common beamline control systems (EPICS and TANGO), motor controllers (Newport, PI and Smaract) and I/O cards (Agilent and NI). Implementations of Pixelator are also running on the HERMES (Soleil) and MAXYMUS (BESSY II) STXM beamlines, and we invite other STXMs to join the Pixelator community. Recent updates to Pixelator have focused on efficiency and reliability, as well as the development of a Python module for scripting experiments. The Python module interacts with the Pixelator server via the same JSON/zero message queue interface as used by the Pixelator GUI and so has access to all functions. The sub-100 ps pump-probe dynamics and in-situ transport measurements discussed by Finizio et al $[20,21]$ is integrated as a detector in Pixelator. All data are stored in NeXus-compliant HDF5 files,[22] including the interferometer positions and ring current values on a pixel-by-pixel basis, allowing accurate normalization and re-gridding of images. In order to tightly integrate complex user-experiments into the STXM measurements, Pixelator can be easily configured to record user-defined data sources on a pixel-by-pixel basis via analog or counter inputs, as well as digital channels such as EPICS. We are currently implementing the insertion of thumbnail images into the HDF5 files (following the XMP standard,[23] commonly known for including metadata in graphics files), together with file browser plugins for the major operating systems as a convenience for users sorting through large numbers of data files.

Recent work by Rösner et al [24,25] has demonstrated $7 \mathrm{~nm}$ spatial resolution at the bend-magnet based PolLux STXM, with the aid of a newly installed Z-axis interferometer. This third interferometer axis is fully integrated into the Pixelator software and provides feedback for position stabilization of the sample (relative to the zoneplate) along the beam axis in order to cope with the short depth of focus of the high-resolution zoneplates.

PolLux is collaborating on the development of a new generation of sample environmental cells that leverage advanced fabrication technologies to integrate measurement and control devices into a silicon chip. Firstly, we are supporting NanoInsight and the Max-Planck-Institut für Chemische Energie 
Konversion in the development of high temperature, high pressure nano-reactors for catalysis research. Secondly, we are also collaborating with the Atmospheric Chemistry group at PSI to develop a low temperature, high precision cell for research into ice nucleation.

The PolLux STXM continues to provide high performance and reliability to the soft X-ray spectro-microscopy community. We encourage researchers to further incorporate complex sample environments into their experiments and take advantage of the integration opportunities provided by the Pixelator experiment control software.[26]

\section{References:}

[1] J Raabe et al, Rev. Sci. Instrum. 79 (2008) 113704.

[2] U Flechsig et al, AIP Conf. Proc. 879 (2006) p.505.

[3] U Frommherz et al, AIP Conf. Proc. 1234 (2010) p.429.

[4] K Witte et al, J. Phys. Chem. B 122 (2018) p.1846.

[5] C Xie et al, Adv. Energy Mater. 8 (2018) 1702857.

[6] X Du et al, Macromol. 50 (2017) p.2415.

[7] J Farinhas et al, Org. Electron. 41 (2017) p.130.

[8] R Janneck et al, Adv. Mater. 29 (2017) 1703864.

[9] S Woo et al, Nat. Commun. 9 (2018) p.959.

[10] D Maccariello et al, Nature Nanotechnol. 13 (2018) p.233.

[11] S Finizio et al, Phys. Rev. B 96 (2017) 054438.

[12] F Fritz et al, Nanotechnology 28 (2017) 435703.

[13] M Baumgartner et al, Nat. Nanotechnol. 12 (2017) p.980.

[14] PA Alpert et al, Sci. Rep. 7 (2017) 12693.

[15] I Pignatelli et al, Geochim. Cosmochim. Ac. 209 (2017) p.106.

[16] J Miot et al, Chem. Geo. 434 (2016) p.28.

[17] S Bonneville et al, Environ. Sci. Technol. 50 (2016) p.5589.

[18] B Watts et al, J. Instrum. 13 (2018) C04001.

[19] https://www.psi.ch/microspec/pixelator

[20] S Finizio et al, XRM2018 (2018) 015243.

[21] S Finizio et al, XRM2018 (2018) 013869.

[22] B Watts et al, AIP Conf. Proc. 1696 (2016) 020042.

[23] ISO 16684-1:2012.

[24] B Rösner et al, Microelectronic Engineering 191 (2018) p.91.

[25] B Rösner et al, XRM2018 (2018) 015376.

[26] The PolLux end station was financed by the German Minister für Bildung und Forschung (BMBF) through contracts 05KS4WE1/6 and 05KS7WE1. 